\title{
ACTUALIZACIONES
}

\section{MICROBIOTA INTESTINAL Y CIRUGÍA BARIÁTRICA}

\section{GUT MICROBIOTA AND BARIATRIC SURGERY}

\author{
María Graciela Álvarez ${ }^{1}$
}

\section{RESUMEN}

En los últimos años, el estudio de la microbiota intestinal emergió como un nuevo factor ambiental en la patogenia de la obesidad y la diabetes tipo 2 (DM2), enfermedades complejas, heterogéneas, crónicas y progresivas. Los tratamientos convencionales de la obesidad mórbida fracasan, y actualmente se considera a la cirugía bariátrica como el único tratamiento efectivo de la obesidad severa, definida como IMC >40 ó IMC >35 asociado a comorbilidades. Los mecanismos de restricción y malabsorción fueron propuestos inicialmente como responsables del descenso de peso y la mejoría metabólica observados luego de la cirugía bariátrica, sin embargo, no pueden explicar la rápida remisión de la DM2 con el procedimiento quirúrgico. Aunque se investigan intensamente, no se conocen con exactitud los mecanismos causales de los beneficios metabólicos. Se postulan los siguientes: cambios neuroendocrinos asociados al redireccionamiento del flujo de nutrientes, modificaciones del vaciamiento gástrico y motilidad intestinal, cambios en la conducta alimentaria, remodelamiento del asa de Roux, alteración de la circulación enterohepática de ácidos biliares, gluconeogénesis intestinal y modificaciones en la microbiota intestinal. Se considera a la microbiota un eslabón entre la dieta rica en grasas, la inflamación sistémica y la homeostasis de la glucosa. Algunos estudios en animales y en humanos sugieren que los cambios en la composición de la microbiota pueden contribuir a los beneficios metabólicos observados luego del bypass gástrico en-Y-de Roux (BGYR).

Palabras clave: obesidad, cirugía bariátrica, diabetes tipo 2 , microbiota

Revista de la Sociedad Argentina de Diabetes 2015; Vol. 49 (32-40)

\footnotetext{
${ }^{1}$ Clínica Médica. Especialista en Nutrición con orientación en Obesidad. Docente de la Universidad Favaloro. Médica Clínica y Nutricionista del Centro de Estudio y Tratamiento de la Obesidad Severa (CETOS)
}

Contacto del autor: María Graciela Álvarez

E-mail: alvarez_mariagraciela07@yahoo.com.ar

Correspondencia: Santa Fe $39967^{\circ}$ piso Depto. 81

(C1425BHO), CABA, Argentina. Tel.: (011) 4831-8394

Fecha de trabajo recibido:18/05/15

Fecha de trabajo aceptado:11/02/15

Conflictos de interés: ninguno que declarar

\begin{abstract}
In the last years, the study of gut microbiota has emerged as a new environmental factor in obesity and type 2 diabetes pathogeny, complex, heterogeneous, chronic and progressive diseases. Conventional treatments fail in morbid obesity, and bariatric surgery is considered at present as the only effective treatment in severe obesity, defined as $B M I>40$, or $B M I>35$ associated with comorbidities. The malabsorption and restriction mechanisms, initially proposed as the responsible for the weight loss and metabolic amelioration observed after bariatric surgery, do not adequately explain the quick remission of type 2 diabetes seen with the surgical procedure. The causal mechanisms are not exactly known, in spite of the profuse research on the matter. The following factors have been postulated: neuroendocrine changes associated with the re-direction of the flow of nutrients, changes in gastric emptying and intestinal motility, changes in feeding behavior, remodeling of Roux loop, changes in the enterohepatic circulation of bile acids, intestinal gluconeogenesis, and alterations in gut microbiota. Gut microbiota is seen as a link between dietary intake, systemic inflammation, and glucose homeostasis. Some studies in animal models and in humans suggest that microbiota composition modification may contribute to the metabolic benefits observed after the Roux-en-Y gastric bypass.
\end{abstract}

Key words: obesity, bariatric surgery, type 2 diabetes, microbiota Revista de la Sociedad Argentina de Diabetes 2015; Vol. 49 (32-40)

\section{INTRODUCCIÓN}

La obesidad y la DM2 resultan de la interacción entre factores genéticos y ambientales, y entre los últimos, las bacterias intestinales parecen tener un rol importante. Si bien el aumento y la calidad de la dieta y la falta de actividad física son los factores causales clásicos de la enfermedad metabólica, éstos no logran explicar la creciente prevalencia de la obesidad y la diabetes, por lo que se investigan otros factores ambientales, incluidos los cambios en la microbiota. La manipulación de la microbiota, todavía en etapas de experimentación, brindaría nuevas posibilidades terapéuticas.

Existen diferentes técnicas quirúrgicas para el tratamiento de la obesidad severa y las alteraciones metabólicas asociadas; las más usadas en la actualidad son el BGYR y la gastrectomía vertical 
en manga o manga gástrica ${ }^{1,2}$. Si bien hay diferencias anatómicas entre ambas, con las dos técnicas se alcanza una disminución aproximada del $30 \%$ del peso y la remisión de la DM2 en el largo plazo en la mayoría de los pacientes ${ }^{3}$.

Un reciente estudio demostró que hasta un tercio de los pacientes continúa en remisión de la DM2 luego de 15 años de la cirugía 4 . El BGYR mejora la insulinorresistencia antes de que se alcance un descenso de peso significativo, por lo que se asume que existen factores independientes del peso en la mejoría metabólica ${ }^{5}$. No se conocen con exactitud los mecanismos a través de los cuales el BGYR logra la remisión de la DM2. La Figura 1 muestra en forma esquemática los mecanismos de acción propuestos. A pesar de que se investigan intensamente los cambios neurohormonales post cirugía -GLP-1, PYY, grelina- no pudo hallarse un factor responsable único de los beneficios metabólicos. Las interpretaciones mecánicas o neuroendocrinas no pueden explicar los resultados alcanzados con la cirugía, por lo que el foco de la investigación se derivó hacia otros mecanismos moleculares potenciales. Recientemente dos áreas de investigación se focalizaron en los cambios de la microbiota intestinal y del metabolismo de los ácidos biliares como factores que contribuyen a los beneficios metabólicos postquirúrgicos. Resulta interesante que ambos mecanismos están interrelacionados, los ácidos biliares sufren un metabolismo transgénico en la circulación entero-hepática y a su vez la concentración de ácidos biliares modula el sobrecrecimiento bacteriano ${ }^{6}$.

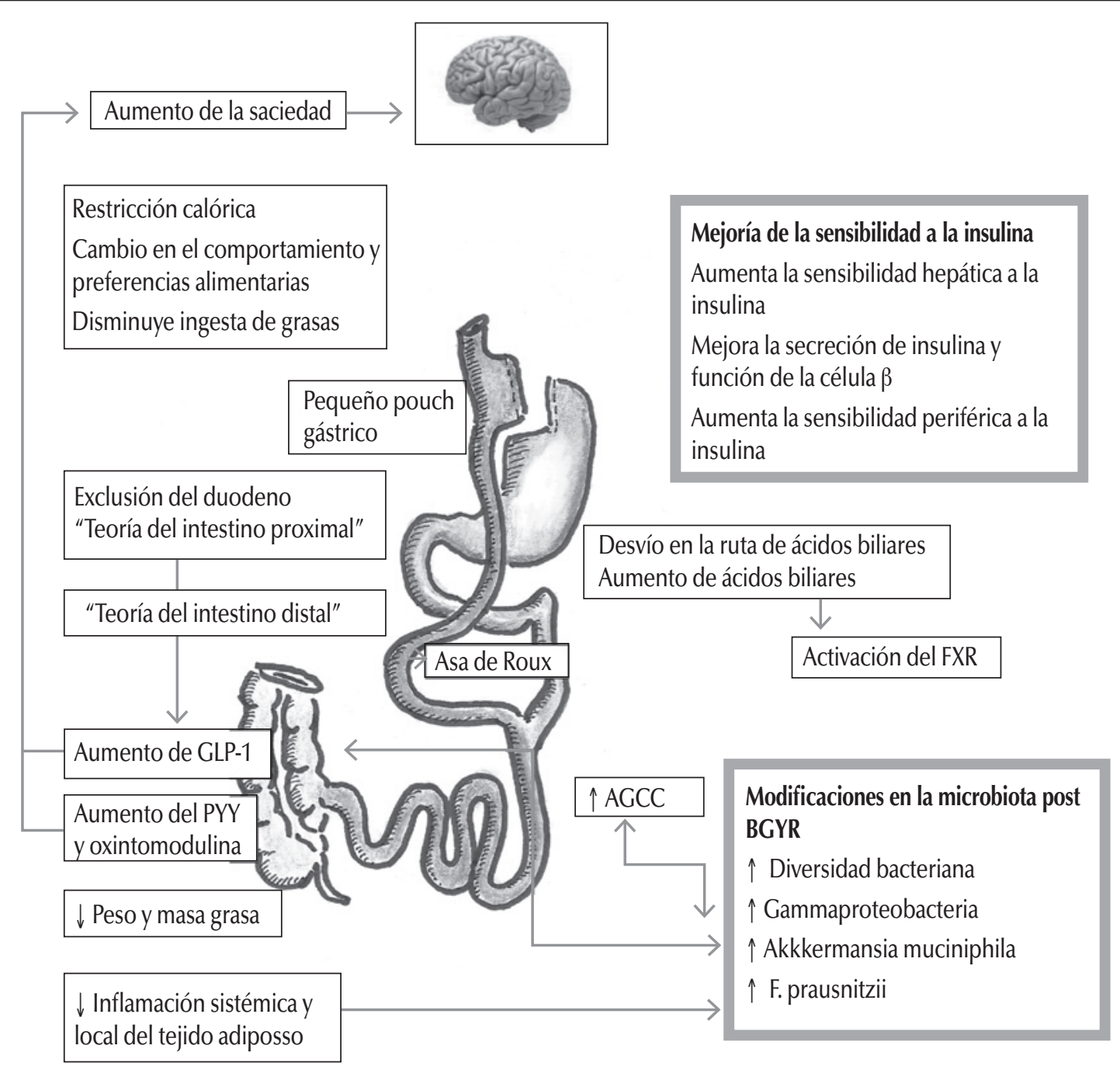

Figura 1: Mecanismos de acción propuestos del BGYR en la mejoría del metabolismo glucémico. Existen simultáneamente cambios en la configuración intestinal con alteración de la circulación de nutrientes, cambios neurohormonales y modificaciones de la microbiota. Adaptado de Aron-Wisnewsky y Clement. Curr. Atheroscler. Rep. 16:454, 201458. 


\section{Microbiota intestinal}

Cada hábitat del cuerpo tiene un grupo único de microorganismos denominado microbiota. El intestino contiene $10^{14}$ bacterias -la mayor densidad microbiana de cualquier hábitat-y más de 1.000 especies?, de las cuales el $95 \%$ vive en el colon ${ }^{8,9}$. La masa de bacterias en la materia fecal representa el 1-3\% del peso corporal y es equivalente al tamaño del hígado $^{10,11}$. El microbioma -los genomas colectivos de la microbiota- supera 100 a 150 veces el genoma humano y fue evolucionando junto a los seres humanos durante miles de años, expandiendo su capacidad fisiológica y metabólica, ahorrando así al huésped la necesidad de evolucionar en vías metabólicas específicas $^{12}$. El microbioma es esencial para la salud y el bienestar; actúa en la función de barrera intestinal, protege contra ciertos patógenos, participa en el desarrollo de las microvellosidades intestinales y en la transformación de ácidos biliares conjugados y de complejos de oxalato, produce vitaminas (B12 y K), colabora en la extracción de energía de la dieta a través de la fermentación de la fibra y es un importante regulador del desarrollo de la inmunidad innata y adaptativa ${ }^{13}$. Las bacterias intestinales producen moléculas con actividad inmunomoduladora (ácidos nucleicos y oligonucleótidos bacterianos, proteínas y péptidos, polisacáridos, neurotransmisores, ácidos biliares, ácidos grasos de cadena corta, productos de degradación de la colina, lípidos mediadores de la inflamación, y endocannabinoides) que pasan a la sangre a través de la circulación enterohepática o por aumento de la permeabilidad de la mucosa intestinal ${ }^{14}$. Mientras que algunas bacterias son beneficiosas y producen vitaminas y antioxidantes, otras pueden dañar el ADN, afectar el SNC o el sistema inmune y ser perjudiciales para el metabolismo.

La microbiota conforma un órgano virtual, exteriorizado, que participa en el metabolismo y en el balance energético a través de diversos mecanismos y funciones. Una función importante se relaciona con el aumento en la extracción de energía de la fibra alimentaria. La fermentación de la fibra y de los polisacáridos complejos no digeribles libera monosacáridos y ácidos grasos de cadena corta (AGCC): acetato, propionato y butirato. Éstos son sustratos energéticos, equivalentes al $10 \%$ de la energía de la dieta y también son importantes reguladores metabólicos ${ }^{15}$. Los AGCC activan receptores ligados a la proteína G: GPR41 y GPR43; a nivel de las células enteroendocrinas del íleon distal y colon estimulan la liberación de PYY y GLP-1, y a nivel del adipocito favorecen la lipogénesis, inducen la producción de leptina e inhiben la lipólisis ${ }^{15}$. El butirato, además de ser nutriente para los colonocitos, interviene en la función de barrera intestinal incrementando las uniones estrechas entre las células epiteliales a través de cambios en la localización y distribución de zonula occludens-1; estos complejos de unión son macromoléculas formadas por proteínas trasmembrana, como la claudina y occludina. Las uniones estrechas o zonula occludens bloquean la translocación de lipopolisacáridos (LPS) al torrente sanguíneo, potentes mediadores de la inflamación que conducen al aumento de la insulinorresistencia, de la masa grasa total y hepática, y del peso corporal. Así las bacterias productoras de butirato previenen la "endotoxemia metabólica" y evitan que los complejos LPS/CD14 estimulen la infiltración de macrófagos en el tejido adiposo y produzcan inflamación local y sistémica ${ }^{16,17}$. El butirato, además, puede aumentar la actividad mitocondrial en roedores, la regulación hormonal y activar la expresión de genes de la gluconeogénesis intestinal influyendo en el metabolismo de la glucosa y en la ingesta ${ }^{18}$. En modelos animales, el butirato afecta los niveles de serotonina intestinal y aumenta los transportadores de serotonina en el hipotálamo. La serotonina se relaciona con la permeabilidad intestinal además de ser un importante regulador de la ingesta y la saciedad ${ }^{19}$.

Resumiendo: la producción de AGCC no sólo aumenta la extracción de energía de la dieta, sino que también modula la inflamación y la respuesta inmune del huésped, estimula la producción de PYY y GLP-1, refuerza el eje intestino-cerebro en el control del apetito y mejora la función de la célula $\beta^{20}$.

La mayoría de las bacterias intestinales pertenece a 7 phyla o divisiones: el $90 \%$ pertenece a los Firmicutes y Bacteroidetes y el resto a Actinobacteria, Proteobacteria, Verrucomicrobia, Fusobacteria y Cyanobacteria ${ }^{21}$. También están presentes archeas metanogénicas y gran cantidad de virus, sobre los que se dispone de menos información. Las bacterias de cada phylum bacteriano se clasifican en géneros y especies, por ejemplo al phylum Firmicutes pertenecen 200 géneros y a cada género decenas a cientos de especies. Los investigadores buscan identificar las bacterias particulares a nivel de la especie que están involucradas en el desarrollo de la enfermedad metabólica. La Tabla 1 muestra la clasificación taxonómica de las bacterias intestinales. 


\section{Phylum}

Firmicutes

250 géneros

Bacteroidetes

20 géneros

Proteobacteria

Verrucomicrobia

Actinobacteria

\section{Cyanobacteria}

\section{Fusobacteria}

Dominio Archaea

Euryarchaeota

\section{Género}

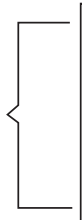

Lactobacillus

Bacilli: Bacillus, Listeria

Ruminococcus/Enterococcus/Peptostreptococcus

Clostridium: C. dificcile, C. tetani, C. botulinum, Roseburia

Mycoplasma/Faecalobacterium prausnitzii/Eubacterium

\section{Bacteroides/Prevotella}

E. Coli, H. pylori, K.E.S., Pseudomonas, Salmonella, Shigella, Vibrio cholerae

Akkermansia muciniphila

Bifidobacterium/Actinomyces/Micrococcus

Bifidobacterium/Actinomyces/Micrococcus

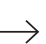

$\longrightarrow$

$\longrightarrow$

Methanobrevibacter smithii

Tabla 1: Clasificación taxonómica de las bacterias intestinales. La comunidad bacteriana varía cuali y cuantitativamente a lo largo del tubo digestivo por factores del huésped $(\mathrm{pH}$, tiempo de tránsito, ácidos biliares, enzimas digestivas y mucus), factores extrínsecos (nutrientes, medicamentos) y factores bacterianos (capacidad de adhesión, enzimas y capacidad metabólica). Se enumeran algunos géneros conocidos a manera de ejemplo.

Tanto factores genéticos, epigenéticos, como ambientales configuran la flora única de cada individuo después de la colonización inicial al nacer; la microbiota se mantiene estable a partir del segundo o tercer año de vida ${ }^{22}$. Posteriormente la microbiota puede modificarse por la calidad de la dieta, su contenido calórico, los fármacos (por ejemplo, los antibióticos y la metformina), los pre y probióticos, y el envejecimiento. El uso de antibióticos en las primeras etapas de la vida tiene mucha importancia y puede relacionarse con la disminución de la diversidad bacteriana, mayor cantidad de masa grasa y riesgo de desarrollar obesidad en la vida adulta ${ }^{23}$.

No es posible definir una microbiota normal, cada individuo tiene un patrón único de composición bacteriana. Esta variabilidad interindividual en la composición de la microbiota (sólo se comparte el $0,9 \%$ de las bacterias en el intestino) probablemente se deba a la gran redundancia funcional, dado que comunidades diferentes de bacterias convergen hacia las mismas funciones. Se iden- tifican familias de genes: un "core microbioma" a nivel funcional; existe la posibilidad que desviaciones de ese "core" o disbiosis se asocien a estados patológicos como la obesidad y la DM2 ${ }^{24}$.

La estratificación en riqueza de genes microbianos o cantidad de genes del microbioma es predictiva del estatus metabólico e inflamatorio del huésped y puede considerarse como un nuevo biomarcador ${ }^{25-27}$. Estudios metagenómicos, el Proyecto Microbioma Humano del NIH en Estados Unidos y el metaHit (Metagenomic of the human intestinal tract) en Europa demostraron que la menor diversidad bacteriana se asocia con IMC más altos, dislipidemia, alteraciones del metabolismo de la glucosa, insulinorresistencia e inflamación sistémica de bajo grado $^{21,28}$. Las intervenciones nutricionales modifican la microbiota y mejoran el perfil metabólico, pero parecen ser menos efectivas en los individuos con menor riqueza bacteriana. Algunos grupos postulan que las alteraciones en la composición bacteriana y la menor riqueza en genes bacterianos diferencian a 
los obesos metabólicamente sanos de los obesos con enfermedad metabólica. El promedio de los adultos de Estados Unidos tiene un 15-20\% menos de riqueza bacteriana que los habitantes nativos de Malawi (África) o Venezuela; este hecho sugiere que la dieta occidental, rica en grasas y pobre en fibra, conduce a la pérdida de la microbiota en número de genes y diversidad bacteriana ${ }^{28}$. La restricción calórica durante seis semanas se asocia con aumento del número y diversidad de genes bacterianos ${ }^{29}$. La cirugía bariátrica también aumenta la biodiversidad de la microbiota ${ }^{30-33}$. Por el contrario, con el envejecimiento disminuye la diversidad bacteriana y aumenta el nivel inflamatorio ${ }^{29}$.

\section{Microbiota intestinal y obesidad}

Las personas con sobrepeso y obesidad tienen una composición bacteriana distinta en comparación con la de sujetos de peso normal; una "microbiota obesa" que es más eficiente en la extracción de energía de la dieta. Los obesos tienen menor diversidad bacteriana y mayor proporción de Firmicutes en relación a los Bacteroidetes, aunque no todos los resultados son consistentes con estos datos, posiblemente debido a diferencias en las técnicas utilizadas ${ }^{32}$. Presentan aumento de la expresión genética de las vías metabólicas que favorecen la extracción de energía, y tienen menos energía residual en la materia fecal ${ }^{34}$. Por otro lado, la microbiota modula la inflamación y la insulinorresistencia, y contribuye al aumento de la masa grasa y del peso corporal ${ }^{35}$. Los primeros trabajos que relacionaron la microbiota intestinal con la obesidad fueron realizados en modelos animales. En el año 2004, Friedrick Bäckhed et al. observaron que el trasplante de microbiota a ratones germ-free (sin gérmenes, axénicos o gnotobióticos) provocó aumento del peso y de la insulinorresistencia, con incremento de más del $60 \%$ de la masa grasa, a pesar de la disminución de la ingesta ${ }^{36}$. Los ratones germ-free tienen menos SGLT-1 en el epitelio intestinal y absorben menos glucosa. La microbiota inhibe el FIAF (fasting-induced adipose factor) a nivel del intestino, con lo cual aumenta la LPL, favorece la captación de AGL y el depósito de TGC en el adipocito y conduce a la hipertrofia adipocitaria. El trasplante de microbiota aumenta las vellosidades del intestino promoviendo el transporte de monosacáridos por la circulación portal hacia el hígado, produce aumento de la glucemia y la insulinemia en paralelo al incremento de la masa grasa medida por DEXA. Provoca aumento de las enzimas claves y de los factores de transcripción necesarios para la lipogénesis de novo (Acc-1, sintasa de ácidos grasos, SREBP-1 y ChREBP). Por otro lado, los ratones germ-free son resistentes a la obesidad inducida por la dieta, sugiriendo la existencia de algún factor mediado por la microbiota en la patogenia de la obesidad $^{36}$. Este grupo demostró por primera vez que la microbiota, un factor ambiental, modula el metabolismo y contribuye a la regulación del almacenamiento energético del huésped ${ }^{36,37}$. En otro estudio, ratones genéticamente idénticos alimentados con igual dieta rica en grasa exhibieron fenotipos metabólicos distintos atribuibles a diferencias en la microbiota ${ }^{37,38}$.

En el año 2005, el equipo de Jeffrey Gordon publicó que los ratones ob/ob tienen 50\% más Firmicutes y $50 \%$ menos Bacteroidetes que los ratones de peso normal, y desarrolló la hipótesis de que los Firmicutes tienen mayor capacidad para extraer energía de la dieta y conducen de este modo a la ganancia de peso $^{29}$. Posteriormente, estudios en roedores y en humanos obesos demostraron que la relación Firmicutes/Bacteroidetes se invierte con la restricción calórica y la disminución de peso ${ }^{30,33}$.

Se observó que los ratones obesos tienen menor concentración de Akkermansia muciniphila, una bacteria del phylum Verrucomicrobia que se alimenta de la mucina de la pared intestinal y se relaciona con la función de barrera intestinal. Se asocia con mayor secreción de GLP-1, disminución de la endotoxemia metabólica, mejoría de la insulinorresistencia y menor inflamación sistémica y local a nivel del tejido adiposo ${ }^{39}$. Es interesante que el tratamiento con metformina se vincula con el aumento de la concentración de Akkermansia en la microbiota de ratones con obesidad inducida por la dieta ${ }^{40}$.

Los beneficios metabólicos pueden transferirse a través de la microbiota. Vanesa Ridaura et al. trasplantaron materia fecal de cuatro pares de mellizas discordantes delgadas/obesas a ratones germ-free, y observaron que la microbiota de las mujeres obesas transmitía el fenotipo obeso y el de las delgadas, el delgado ${ }^{41}$. El fenotipo obeso o delgado se transmitió a través de la microbiota sin relación con cambios en la ingesta o la inflamación. En otro trabajo se trasplantó microbiota de hombres adultos sanos a pacientes con DM2 y síndrome metabólico, y a las seis semanas se obtuvo mejoría de la insulinosensibilidad medida por clamp euglucémico, aumento de la diversidad 
de la microbiota e incremento en la concentración de bacterias productoras de butirato ${ }^{42}$.

\section{Microbiota y diabetes tipo 2}

La obesidad y la DM2 comparten una característica en común que es la inflamación de bajo grado en tejidos involucrados con la regulación del metabolismo, tales como el hígado, el tejido adiposo y el músculo estriado ${ }^{43}$. El aumento de peso con la hipertrofia adipocitaria, el cambio de secreción de adipoquinas hacia un perfil más inflamatorio y la atracción de macrófagos hacia el tejido adiposo, sería el factor inicial de la cascada inflamatoria. Modulando la inflamación, la microbiota intestinal puede contribuir en la patogenia de la obesidad y la DM2. Se propusieron varios mecanismos que relacionan a la microbiota con la insulinorresistencia y la DM2: la endotoxemia metabólica, las modificaciones en la secreción de incretinas y la producción de butirato ${ }^{43}$.

La dieta rica en grasas aumenta la proporción de bacterias gram negativas que contienen lipopolisacáridos (LPS), endotoxinas de la pared bacteriana, el cambio en la concentración de bacterias o en la permeabilidad intestinal que aumenta la endotoxemia metabólica y correlaciona en forma directa con el grado de insulinorresistencia ${ }^{44}$. Este rol causal fue demostrado con la infusión de LPS a roedores alimentados con dieta normal, que provoca aumento de la insulinorresistencia hepática, trastornos de la tolerancia a la glucosa e incremento de peso y masa grasa. Los LPS se unen a los receptores CD14/TLR4 de la pared de los macrófagos estimulando la secreción de citoquinas inflamatorias; la infusión de LPS a ratones knockout para CD14/TLR4 no condujo al desarrollo de obesidad o DM2. Los LPS también promueven la expresión de NF-kB y de MAPK en los adipocitos ${ }^{45}$.

La modulación de la microbiota con prebióticos aumenta la concentración de Bifidobacterium spp y se acompaña de un aumento en la secreción de GLP-1 y PYY46. También aumenta la secreción de GLP-2, y ésta se asocia con mayor expresión de zonula occludens-1 que mejora la función de barrera intestinal y disminuye la concentración de LPS en plasma ${ }^{47}$.

Las personas con DM2 tienen menor cantidad de bacterias productoras de butirato en su microbiota; el análisis genético de las funciones bacterianas muestra un aumento de las funciones relacionadas con la respuesta al estrés oxidativo intestinal ${ }^{48}$.

En pacientes con DM2 o con intolerancia a la glucosa se observa mayor proporción de Bacteroidetes y de Proteobacteria, así como también menor número de bacterias antiinflamatorias productoras de butirato: Roseburia spp y Faecalibacterium spp, en comparación con sujetos sanos ${ }^{49,50}$. También se describió menor concentración del $\mathrm{F}$. prausnitzii en obesos mórbidos con DM2 versus obesos sin DM2, y en diabéticos comparados con sujetos con intolerancia a la glucosa. El F. prausnitzii bloquea al factor nuclear kappa B (FNkB) y secreta metabolitos antiinflamatorios ${ }^{51}$.

En un estudio en animales, Liou et al. trasplantaron materia fecal de ratones operados de BGYR a ratones diabéticos, obteniendo como resultado disminución de peso y mejoría del metabolismo glucémico y lipídico asociados a cambios en la composición de la microbiota productora de butirato ${ }^{52}$.

\section{Microbiota y cirugía bariátrica}

EI BGYR modifica la microbiota hacia una más parecida a la de los sujetos de peso normal; estos cambios en la microbiota podrían contribuir a la mejoría de la insulinorresistencia y del metabolismo glucémico obtenidos con la cirugía ${ }^{53,54}$.

Luego del BGYR existen modificaciones en la composición bacteriana a nivel de phylum, disminuyen los Firmicutes y aumentan los Bacteroidetes, junto con un aumento marcado de la diversidad bacteriana ${ }^{55}$. Otro hallazgo sistemático es el aumento de Gammaproteobacteria (Escherichia) y Verrucomicrobia (Akkermansia) ${ }^{52}$, que correlacionan con el descenso de peso y la mejoría de factores inflamatorios como la disminución de la infiltración de macrófagos en el tejido adiposo y de la formación de estructuras en corona que rodean a los adipocitos muertos ${ }^{56}$. Estudios en animales ${ }^{53}$ y en humanos ${ }^{54,57}$ muestran disminución de Firmicutes luego de la cirugía bariátrica, sin embargo, en pacientes diabéticos operados de BGYR se observa a nivel de especies, aumento del Faecalobacterium prausnitzii (perteneciente al phylum de los Firmicutes) asociado a la mejoría de la insulinorresistencia y disminución de factores inflamatorios como IL-6 y PCRus ${ }^{30}$.

Estas asociaciones observadas no permiten aclarar si los cambios en la microbiota son causa o consecuencia de la mejoría metabólica ${ }^{58}$. Resulta complejo establecer una relación de causalidad entre los cambios en la microbiota y el beneficio metabólico observado con la cirugía bariátrica, ya que cambian simultáneamente la dieta, el pH, la disponibilidad de 
nutrientes intestinales, los niveles de sales biliares y la secreción de hormonas intestinales. En el BGYR aumenta el $\mathrm{O} 2$ disuelto y se eleva el $\mathrm{pH}$, además de modificarse la disponibilidad de nutrientes ${ }^{57}$; se describieron extensamente los cambios hormonales post BGYR que no serán tratados aquí. El uso de ATB preoperatorios puede provocar cambios transitorios en la microbiota ${ }^{58}$. En roedores operados de GBYR se observó una disminución significativa de la actividad de DPPIV a nivel intestinal y sistémico, junto con los cambios de la microbiota ${ }^{57}$.

Un estudio sugirió un rol más directo de la microbiota en los beneficios metabólicos de la cirugía, el trasplante de materia fecal de roedores operados de BGYR a animales germ free. Los ratones receptores disminuyeron de peso y perdieron el $5 \%$ de la masa grasa, en comparación con los receptores de microbiota de ratas con operación simulada o de un tercer grupo de ratas con operación simulada y restricción calórica ${ }^{52}$. Este trabajo insinúa que los cambios en la microbiota son atribuibles a la reconfiguración intestinal más que a la restricción calórica y al descenso de peso. La disminución de la masa grasa sin cambios en la ingesta sugiere que la microbiota disminuye la eficiencia en extracción de energía de la dieta, o que produce señales que afectan el gasto energético y el metabolismo lipídico. En los ratones receptores se observó una población de bacterias más semejante a la de individuos delgados junto con el aumento de ácidos biliares ${ }^{52}$. La bacteria predominante observada fue la A. muciniphila ${ }^{52,58}$. El análisis de los AGCC en la materia fecal demostró aumento de propionato y disminución de acetato, mientras que no hubo cambios en la excreción de butirato $^{52,59}$. En otro estudio en ratones operados de manga gástrica se observó un aumento de 12 veces de Roseburia asociado a la disminución del peso y mejoría de la insulinosensibilidad, a pesar de recibir una dieta rica en grasas $^{60}$.

La cirugía produce cambios significativos en la composición de la microbiota; ésta podría actuar por mecanismos indirectos a través de los ácidos biliares. La modulación de los ácidos biliares por la microbiota puede estimular el eje intestino-cerebro-hígado, regular la saciedad y mejorar el metabolismo de la glucosa ${ }^{61}$.

\section{Microbiota y ácidos biliares}

Se advirtió aumento del nivel de ácidos biliares luego del BGYR en humanos ${ }^{60,62,63}$ y luego de la manga gástrica en roedores ${ }^{64}$. Los niveles de ácidos biliares se duplican con el BGYR en comparación con la banda gástrica ajustable, técnica con la cual la mejoría metabólica depende más del descenso de peso que de otros mecanismos independientes del peso ${ }^{65}$.

Además de actuar en el metabolismo del colesterol, las grasas y las vitaminas liposolubles, los ácidos biliares son importantes moléculas de señalización, se comportan como hormonas sobre receptores nucleares y de membrana en las células del hígado y del intestino, e intervienen en el metabolismo de la glucemia y de los triglicéridos. Existen transportadores y receptores de ácidos biliares en plasma, hígado, riñón y corazón, lo cual sugiere un rol global de señalización fuera del circuito enterohepático ${ }^{65}$.

Los ácidos biliares son metabolitos transgénicos sintetizados por el hígado y liberados a la bilis. Su síntesis está regulada por un feedback negativo: los ácidos biliares activan el receptor nuclear farnesoide $X$ (FXR) en los enterocitos del íleon para liberar FGF-15/19 (factor de crecimiento fibroblástico), éste actúa sobre los hepatocitos suprimiendo su síntesis66,67. El 95\% de los ácidos biliares regresa al hígado por la circulación enterohepática y el resto es deconjugado por la microbiota y eliminado como ácidos biliares secundarios que tienen acción antibacteriana a nivel del colon dependiendo de su concentración. En ratones germfree existe un aumento del $25 \%$ del colesterol y de los ácidos biliares conjugados en sangre, mientras que disminuye su excreción fecal ${ }^{68}$.

La señal del FXR regula el metabolismo lipídico, de la glucosa y de los ácidos biliares. A nivel hepático, el FGF15 compite con el FXR y puede estar involucrado en la síntesis diferencial de dos ácidos biliares primarios, ácido cólico y muricólico en ratones, con diferencias en la solubilidad de colesterol en las micelas (uno es más hidrofílico y el otro más anfipático) modulando así la absorción y producción de colesterol por el hígado. También existe FXR en la célula beta, lo cual sugiere una regulación en la homeostasis de la glucosa ${ }^{67,68}$.

La microbiota influye por varios mecanismos en el metabolismo de los ácidos biliares: aumenta la expresión del ASBT (apical sodium dependent bile acid transporter) y del FGF15. Aparentemente el FXR es desactivado en ausencia de microbiota ileal.

La microbiota puede influir indirectamente en la esteatosis hepática y la lipoperoxidación por acción del FXR sobre la secreción de ácidos biliares. Por otro lado, los ácidos biliares secundarios inhi- 
ben la captación de AGL por el hígado y podrían disminuir de esta forma la esteatosis hepática ${ }^{68,69}$.

En un estudio reciente en roedores operados de manga gástrica, los efectos beneficiosos sobre el peso y el metabolismo glucémico fueron abolidos en el grupo knock out para el FXR. La señal del FXR parece ser clave en los cambios de la microbiota, ya que ratones operados de BGYR y que son knock out para el FXR, no muestran una microbiota del fenotipo delgado60.

En roedores se observó que la simple derivación de la bilis hacia el intestino distal a través de un catéter, sin otros cambios en el tubo intestinal, produjo mejoría de la insulinorresistencia y disminución del peso a pesar de ingesta similar, en comparación con ratones con intervención simulada ${ }^{65}$.

Los ácidos biliares también actúan como moléculas de señalización sobre el receptor de membrana TGR5: a nivel del tejido adiposo pardo aumentan el gasto energético, y a nivel de las células $L$ del íleon distal y del colon inducen la secreción de GLP-1. Por último, estimulan la 2-iodo-tironinadeiodinasa que convierte T4 en T3, aumentando el metabolismo basal y el gasto energético $0^{69-71-72}$.

\section{CONCLUSIONES}

Las investigaciones en el área de la microbiota se hallan todavía en etapas incipientes y existen aún datos controvertidos, pero parece ser cada vez más claro que existe un componente bacteriano en la patogenia de la obesidad y la diabetes tipo $2^{15,19}$.

Muchos equipos en el mundo actualmente investigan los mecanismos a través de los cuales la cirugía bariátrica produce la mejoría metabólica observada, es posible que variaciones en la microbiota contribuyan a los resultados beneficiosos de la cirugía. Por el momento, se requieren más estudios para aclarar el rol preciso de la microbiota en la mejoría del metabolismo glucémico; faltan aún datos sobre la duración o estabilidad de los cambios en la microbiota en el largo plazo, la relación con las diferentes técnicas quirúrgicas y la interacción entre los cambios producidos en la microbiota, y el perfil hormonal e inflamatorio del huésped a nivel local y sistémico ${ }^{59,65}$.

\section{REFERENCIAS}

1. Cohen RV, Rubino F, Schiavon C, Cummings DE. Diabetes remission without weight loss after duodenal bypass surgery. Surg. Obes. Relat. Dis. 2012; 8:e66-e68.

2. Nannipieri M, Baldi S, Mari A, et al. Roux-en-Y gastric bypass and sleeve gastrectomy: mechanisms of diabetes remission and role of gut hormones. J. Clin. Endocrinol. Metab. 2013; 98:4391-4399.
3. Buchwald H, Estok R, Fahrbach K, et al. Weight and type 2 diabetes after bariatric surgery: systematic review and meta-analysis. Am. J. Med. 2009; 122:248-256.

4. Sjöström L, Peltonen M, Jacobson P, et al. Association of bariatric surgery with long-term remission of type 2 diabetes and with microvascular and macrovascular complications. JAMA 2014 311:2297-2304.

5. Dixon JB, le Roux C, Rubino F, et al. Bariatric surgery for type 2 diabetes. Lancet 2012; 379: 2300-11.

6. Arble DM, Sandoval D, Seeley RJ. Mechanisms underlying weight loss and metabolic improvements in rodent models of bariatric surgery. Diabetologia 2015; 58:211-220.

7. Cani P, Delzenne N. The gut microbiome as therapeutic target. Pharmacology \&Therapeutics 2011, 130: 202-212.

8. Rodizo MR, Mendoza MC. Identificación bacteriana mediante secuenciación del ARNr 16S: fundamento, metodología y aplicaciones en microbiología clínica. Enferm. Infecccc. Microbiol. Clin. 2004; 22(4):238-45.

9. Ley R, Peterson D, Gordon J. Ecological and evolutionary forces shaping microbial diversity in the human intestine. Cell 2006; 124: 837-84.

10. Cell Editorial: The Lords of the Human Genoma. Eat to live or live to eat? Cell 2013; 155: 5-7.

11. Robles Alonso V, Guarner F. Linking the gut microbiota to human health. British Journal of Nutrition 2013; 109: S21-S26.

12. Cani P, Delzenne N. Gut microflora as a target for energy and metabolic homeostasis. Current Opinion in Clinical Nutrition and Metabolic Care 2007, 10:729-734.

13. Holmes $\mathrm{E}$, et al. Gut microbiota composition and activity in relation to host metabolic phenotype and disease risk. Cell Metabolism 2012, 16: 559-564.

14. Bäckhed F, Manchester JK, Gordon JL. Mechanisms underlying the resistance to diet-induced obesity in germ-free mice. Proc. Natl. Acad. Sci. USA 2007; 104: 070-984.

15. Tilg $H$, Moschen A. Microbiota and diabetes: an evolving relationship. Gut gutjnl-2014; 306928.

16. Cox L, Blaser M. Pathways in microbe-induced obesity. Cell Metabolism 2013; 17: 883-894.

17. Aron-Wisnewsky J, Clement K. The effects of gastrointestinal surgery on gut microbiota: potential contribution to improved insulin sensitivity. Curr. Atheroscler. Rep. 2014; 16:454-465.

18. De Vadder F, Kovatcheva-Datchary P, Goncalves D, et al. Microbiota-generated metabolites promote metabolic benefits via gut-brain neural circuits. Cell 2014;156:84-96.

19. Hartstra A, Bouter $K$, Bäckhed F, et al. Insights into the role of the microbiome in obesity and type 2 diabetes. Diabetes Care 2015; 38:159-165.

20. D'Aversa F, Tortora A, laniro G, et al. Gut microbiota and metabolic syndrome. Intern. Emerg. Med. 2013; 8 (Suppl 1):S11-S15.

21. Qin J, Li R, Raes J, et al. MetaHIT Consortium. A human gut microbial gene catalog established by metagenomic sequencing. Nature. 2010; 464(7285): 59-65.

22. Cox LM, Blaser M. Pathways in microbe-induced obesity. Cell Metab. 2013; 17: 883-894.

23. Trasande L, BlusteinJ, Liu M, et al. Infant antibiotic exposures and early-life body. International Journal of Obesit. 2013; 37: 16-23.

24. Turnbaugh $P$, Gordon J.The core gut microbiome, energy balance and obesity. J. Physiol 587.17, 2009, 4153-4158.

25. Fang S, Evans R. Wealth management in the gut. Nature 2013; 500: 538-539.

26. Le Chatellier $E$, et al. Richness of human gut microbiome correlates with metabolic markers. Nature 2013; 500: 541-549.

27. Cotillard $A$, et al. Dietary intervention impact on gut microbial gene richness. Nature 2013; 500: 585-592.

28. The Human Microbiome Project Consortium. Structure, function and diversity of the healthy human microbiome. Nature 2012; 486: 207-214. 
29. Ley R, Bäckhed F, Turnbaugh P, Gordon J. Obesity alters gut microbial ecology. PNAS 2005; 102 (31) 11070-11075.

30. Furet JP, et al. Differential adaptation of human gut microbiota to bariatric surgery-induced weight loss. Diabetes 2010; 59 : 3049-3057.

31. Ley RE, Turnbaugh PJ, Klein S, Gordon JI. Microbial ecology: human gut microbes associated with obesity. Nature 2006; 444:1022-3.

32. Deweerdt S. Microbiome. A complicated relationship status. Nature 2014; 508: S61-S63.

33. Sweeney TE, Morton JM. The human gut microbiome: a review of the effect of obesity and surgically induced weight loss. JAMA Surg. 2013;148:563-9.

34. Jones D, Liu WW. Do you have the right guts? Obesity and the gutmicrobioma. Bariatric Times 2015; 12: 21-23.

35. Khanna S, Tosh PK. A clinician's primer on the role of the microbiome in human health and disease. Mayo Clin. Proc. 2014; 89:107-114.

36. Bäckhed $F$, et al. The gut microbiota as an environmental factor that regulates fat storage. PNAS 2004; 101 (44): 15718-15723.

37. Turnbaugh PJ, Bäckhed F, Fulton L, Gordon JI. Diet-induced obesity is linked to marked but reversible alterations in the mouse distal gut microbiome. Cell Host Microbe. 2008; 3:213-23.

38. Vrieze A, Holleman F, Zoetendal EG. The environment within: how gut microbiota may influence metabolism and body composition. Diabetologia 2010; 53:606-613.

39. Creely SJ, et al. Lipopolysaccharide activates an innate immune system response in human adipose tissue in obesity and type 2 diabetes. Am. J. Physiology Endocrinology and Metabolism 2007; 292:740-747.

40. Shin NR, Lee JC, Lee HY, et al. An increase in the Akkermansia spp. population induced by metformin treatment improves glucose homeostasis in diet-induced obese mice. Gut 2014;63:727-35.

41. Ridaura V, Faith J, Rey F, et al. Gut microbiota from twins discordant for obesity modulate metabolism in mice. Science $2013 \mathrm{Vol}$. 341: 6150.

42. Vrieze A, Van Nood E, Holleman F, et al. Transfer of intestinal microbiota from lean donors increases insulin sensitivity in individuals with metabolic syndrome. Gastroenterology 2012; 143:913-916.

43. Moreno-Indias I, Cardona F,Tinahones J, et al. Impact of the gut microbiota on the development of obesity and type 2 diabetes mellitus. Frontiers in microbiology 2014: 190: 1-10.

44. Cani $P$, et al. Metabolic endotoxemia initiates obesity and insulin resistance. Diabetes 2007; 56:1761-1772.

45. Cani $P$, et al. Involvement of gut microbiota in the development of low-grade inflammation and type 2 diabetes associated with obesity. Gut Microbes 2012; 3 (4) 279-288.

46. Cani P, Neyrinck M, Fava F, et al. Dietary non-digestible carbohydrates promote L-cell differentiation in the proximal colon of rats. British Journal of Nutrition 2007;98: 32-37.

47. Cani $P$, et al. Selective increases of bifidobacteria in gut microflora improve high-fat-diet-induced diabetes in mice through a mechanism associated with endotoxaemia. Diabetologia 2007; 50:2374-2383.

48. Kashyap P, Farrugia G. Oxidative stress: key player in gastrointestinal complications of diabetes. Neurogastroenterol. Motil. 2011; 23: 111-114.

49. Larsen N, Vogensen FK, van den Berg FW, et al. Gut microbiota in human adults with type 2 diabetes differs from non-diabetic adults. Plos One. 2010;5:e9085.

50. Qin J. A metagenome wide association stydy of gut microbiota in type 2 diabetes. Nature 2012;490:55-60.

51. Karlsson FH, Tremaroli V, Nookaew I, et al. Gut metagenome in European women with normal, impaired and diabetic glucose control. Nature 2013; 498(7452):99-103.
52. Liou AP, Paziuk M, Luevano JM Jr, et al. Conserved shifts in the gut microbiota due to gastric bypass reduce host weight and adiposity. Sci.Transl. Med. 2013;5:178ra41.

53. Li JV, Ashrafian H, Bueter M, Kinross J, Sands C, le Roux CW, et al. Metabolic surgery profoundly influences gut microbial-host metabolic cross-talk. Gut. 2011; 60(9):1214-23.

54. Zhang H, DiBaise JK, Zuccolo A, et al. Human gutmicrobiota in obesity and after gastric bypass. Proc. Natl. Acad. Sci. U S A. 2009; 106(7):2365-70.

55. Kong LC, Tap J, Aron-Wisnewsky J, et al. Gut microbiota after gastric bypass in human obesity: increased richness and associations of bacterial genera with adipose tissue genes. Am J Clin Nutr. 2013; 98(1):16-24.

56. Cancello R, Henegar C, Viguerie N, et al. Reduction of macrophage infiltration and chemoattractant gene expression changes in white adipose tissue of morbidly obese subjects after surgeryinduced weight loss. Diabetes. 2005; 54(8): 2277-2286.

57. Graessler J, Qin $Y$, Zhong H, Zhang H, et al. Metagenomic sequencing of the human gut microbiome before and after bariatric surgery in obese patients with type 2 diabetes: correlation with inflammatory and metabolic parameters. The Pharmacogenomics Journal (2013) 13, 514-522.

58. Aron-Wisnewsky J, Dore J, Clement K. The importance of the gut microbiota after bariatric surgery. Nat. Rev. Gastroenterol. Hepatol. 2012; 9 (10):590-8.

59. Osto M, Abegg K, Bueter M, le Roux CW, Cani P, LutzTA. Rouxen-Y gastric bypass surgery in rats alters gut microbiota profile along the intestine. Physiol Behav. 2013; 119:92-6.

60. Ryan KK, Tremaroli V, Clemmensen C, et al. FXR is a molecular target for the effects of vertical sleeve gastrectomy. Nature. 2014; 509(7499):183-8.

61. Li J, Ashrafian H, Bueter M, et al. Metabolic surgery profoundly influences gut microbial-host metabolic cross-talk. Gut 2011;60:1214-1223.

62. Patti ME, Houten SM, Bianco AC, et al. Serum bile acids are higher in humans with prior gastric bypass: potential contribution to improved glucose and lipid metabolism. Obesity 2009; 17:1671-7.

63. Jansen PL, van Werven J, Aarts, et al. Alterations of hormonally active fibroblast growth factors after Roux-en-Y gastric bypass surgery. Dig. Dis. 2011; 29(1):48-51.

64. Myronovych A, Kirby M, Ryan K, et al. Vertical sleeve gastrectomy reduces hepatic steatosis while increasing serum bile acids in a weight-loss-independent manner. Obesity (Silver Spring). 2014; 22(2):390-400.

65. Arble D, Sandoval D, Seeley RJ. Mechanisms underlying weight loss and metabolic improvements in rodent models of bariatric surgery. Diabetologia 2015; 58:211-220.

66. Aguiar Vallim, et al. Pleiotropic roles of bile acids in metabolism. Cell Metab. 2013; 17: 657-669.

67. Swann J, et al. Systemic gut microbial modulation of bile acid metabolism in host tissue compartments. PNAS 2011; 108 (suppl. 1): 4523-4530.

68. Kohli R, Seeley R. The search for mechanisms underlying bariatric surgery. Nat. Rev. Endocrinol. 2013; 9: 572-574.

69. Kootte RS. The therapeutic potential of manipulating gut microbiota in obesity and type 2 diabetes mellitus. Diabetes, Obesity and Metabolism 14:112-120, 2012.

70. Kugelberg E. Surgery: altered gut microbiota trigger weight loss. Nat. Rev. Endocrinol. 2013;9(6):314.

71. Kuipers F, Bloks VW, Groen AK. Beyond intestinal soap: bile acids in metabolic control. Nat Rev Endocrinol 2014; 10:488-98.

72. Young Min Cho. A gut feeling to cure diabetes: potential mechanisms of diabetes remission after bariatric surgery. Diabetes Metab. J. 2014;38:406-415. 